\title{
Fatores associados ao uso de cadeira de rodas por idosos institucionalizados
}

\author{
Factors associated with the use of wheelchairs \\ by institutionalized elderly people
}

Elysama Fernandes Alves ${ }^{1}$ Poliana Penasso Bezerra ${ }^{1}$

${ }^{1}$ Curso de Graduação em Fisioterapia, Universidade Federal de Santa Catarina. Rodovia Governador Jorge Lacerda no 3201/Km 35,4, Jardim das Avenidas. 88906-072 Araranguá SC Brasil.elysama_alvess@ hotmail.com
Abstract Due to the high prevalence of wheelchair use by institutionalized elderly people, the scope of this research was to verify whether factors involving gender, age, education, length of institutionalization, comorbidities, cognition and functionality are associated with the use of such equipment, as well as ascertain the reasons for use according to the perceptions of the elderly. It is a cross-sectional descriptive study that included 55 elderly people subdivided into G1 (locomotion without assistance) and G2 (wheelchair users). Analysis of the profile in medical records, cognition and functionality by the Mini-Mental State Examination and Barthel index was conducted. Wheelchair users asked about the factors that lead to wheelchair use were recorded: 33 (60\%) of the elderly were in G1 and 22 (40\%) in G2. There were differences between the groups with respect to functionality $(p=0.005)$. The elderly who have not suffered a stroke are less likely to use a wheelchair (OR: 0.09; 95\% CI: 0.02 to 0.36). Elderly wheelchair users reported: fear of falling, pain, tiredness, weakness in the legs, difficulty walking and lack of assistance in walking. Strokes and functionality are associated with wheelchair use. Knowledge of these factors and the reasons reported by the elderly may enable alternatives of prevention to be defined.

Key words Wheelchairs, Homes for the elderly, Aging
Resumo Devido à alta prevalência do uso de cadeira de rodas por idosos institucionalizados, objetivou-se verificar se os fatores sexo, idade, escolaridade, tempo de admissão, comorbidades, cognição e funcionalidade estão associados ao uso deste dispositivo, como também averiguar os motivos que podem levar a essa utilização segundo a percepção dos idosos. Estudo transversal e descritivo, incluídos 55 idosos subdivididos em G1 (locomoção sem auxílio) e G2 (cadeirantes). Análise do perfil através dos prontuários, cognição e funcionalidade pelo Mini-Exame do Estado Mental e Indice de Barthel. Foram questionados 33(60,0\%) cadeirantes no G1 e 22(40,0\%) no G2 sobre os fatores que os levaram à utilização da cadeira de rodas. Houve diferença entre os grupos em relação à funcionalidade ( $p=0,005)$. O idoso que não tem acidente vascular encefálico tem menor chance de utilizar cadeira de rodas (OR:0,09; IC95\%:0,020,36). Idosos cadeirantes relataram: medo de cair, dor, cansaço, fraqueza nas pernas, dificuldades de caminhar e falta de auxílio na deambulação. $O$ acidente vascular encefálico e a funcionalidade estão associados ao uso de cadeira de rodas. O conhecimento destes fatores e dos motivos relatados pelos idosos pode permitir que alternativas de prevenção sejam traçadas.

Palavras-chave Cadeiras de rodas, Instituição de longa permanência para idosos, Envelhecimento 


\section{Introdução}

O último censo demográfico, de 2010, registrou que os idosos acima de 60 anos já representavam $10,8 \%$ da população do país ${ }^{1}$. A estimativa é que a cada ano 650 mil novos idosos sejam incorporados à população brasileira ${ }^{2}$.

A limitação de mobilidade é um problema comum que afeta de $16 \%$ a $20 \%$ da população idosa ${ }^{3,4}$. Nos últimos anos, a adoção de dispositivos de mobilidade tem sido maior do que se poderia esperar, em resultado do envelhecimento populacional ${ }^{4}$. Essa realidade é cada vez mais evidenciada em instituições de longa permanência para idosos ${ }^{5}$.

Dados da Pesquisa Nacional de Saúde da População canadense apontam que de 222.500 idosos residentes em instituição, 109.000 utilizavam cadeira de rodas ${ }^{6}$. Entretanto, poucas pesquisas investigaram os fatores associados ao uso deste dispositivo especificamente. A maioria das análises concentra-se no uso de dispositivos de mobilidade em geral, incluindo, além da cadeira de rodas, bengalas, muletas e andadores ${ }^{7}$. Fatores como sexo, idade, estado civil, escolaridade, recursos socioeconômicos, capacidade funcional e comorbidades associadas, têm sido identifica$\operatorname{dos}^{5,8-10}$.

Fatores como problemas de saúde e diminuição na capacidade funcional estão diretamente associados ao uso de cadeira de rodas por idosos comunitários ${ }^{7}$. Além disso, fatores físicos e sociais podem ter influência no uso deste dispositivo por idosos cadeirantes residentes em instituição de longa permanência ${ }^{11}$.

Todavia, é necessária uma maior compreensão em relação às características dos idosos que utilizam a cadeira de rodas, a fim de que possam ser propostas medidas que visem a superação das limitações funcionais ${ }^{12}$.

Assim, pode-se evidenciar a necessidade de estudos que analisem os motivos que levam ao uso de cadeira de rodas em instituições de longa permanência, pois a prevalência deste dispositivo só tende a aumentar ao longo dos anos. Os escassos estudos disponíveis são voltados apenas para o conhecimento dos fatores, mas estes não propõem medidas que visem à redução da utilização deste dispositivo, a fim de promover maior independência funcional aos idosos.

Este estudo tem como objetivo verificar a associação do sexo, idade, escolaridade, tempo de admissão, comorbidades, cognição e funcionalidade com o uso de cadeira de rodas; averiguar os motivos que podem levar a essa utilização se- gundo a percepção dos idosos, a fim de propor medidas alternativas.

\section{Método}

O presente estudo caracteriza-se por ser um estudo descritivo, transversal, desenvolvido em duas instituições de longa permanência, localizadas no extremo sul de Santa Catarina. A pesquisa foi realizada após aprovação do Comitê de Ética em Pesquisa do Centro de Hematologia e Hemoterapia de Santa Catarina - HEMOSC, estando de acordo com a resolução do Conselho Nacional de Saúde 196/96.

O grupo experimental foi composto por uma amostra de conveniência, não probabilística, de idosos, tendo como critérios de inclusão, residir em instituição de longa permanência, deambular independentemente ou com cadeira de rodas. Foram excluídos aqueles que utilizassem dispositivo auxiliar de locomoção (bengala, muleta e andador) e os que se encontravam acamados.

Diante dos critérios estipulados, a amostra inicial foi composta por 62 idosos. Destes, 7 foram excluídos por utilizarem dispositivo auxiliar de locomoção (bengala, muleta e andador). Ao todo, 55 idosos foram incluídos, sendo subdividos em dois grupos: G1 (locomoção sem auxílio) e G2 (idosos cadeirantes). Dados representados na Figura 1.

Para traçar o perfil dos idosos residentes, foi realizada uma consulta aos prontuários disponíveis nas instituições. Todos os dados foram anexados em uma ficha cadastral: sexo, idade, escolaridade, tempo de admissão e comorbidades, incluindo acidente vascular encefálico (AVE), hipertensão, dislipidemia e diabetes. Para a avaliação da capacidade cognitiva dos idosos foi aplicado o Mini Exame do Estado Mental (MEEM) e o Índice de Barthel para a funcional.

Segundo Brucki et al. ${ }^{13}$, o MEEM é um teste cognitivo breve, composto por itens a respeito de orientações do espaço temporal, registro, memória de curto prazo, atenção, cálculo, linguagem e praxia constitucional, possibilitando uma avaliação concisa do estado mental do paciente. O escore varia de 0 a 30, considerando-se a escolaridade. Para idosos com baixa escolaridade, utilizam-se os seguintes pontos de corte: (a) analfabeto - 13 pontos; (b) baixa escolaridade (1-4 anos)- 13 pontos; (c) média escolaridade (4 a 8 anos) - 18 pontos; (d) alta escolaridade (acima de 8 anos) -26 pontos. Em relação ao MEEM, os idosos foram classificados em duas categorias: sem declí- 
Figura 1. Fluxograma de inclusões e perdas amostrais.

Legenda: G1 (locomoção sem auxílio), G2 (cadeirantes).

nio cognitivo e com declínio cognitivo, de acordo com a pontuação proposta por Brucki et al. ${ }^{13}$.

Mahoney e Barthel ${ }^{14}$ descrevem que o índice de Barthel é um instrumento que avalia o nível de independência do sujeito para a realização de dez atividades básicas de vida: comer, higiene pessoal, uso dos sanitários, tomar banho, vestir e despir, controle de esfíncteres, deambular, transferência da cadeira para a cama, subir e descer escadas. Na versão original a pontuação da escala varia de $0-100$. A pontuação mínima de zero corresponde à máxima dependência para todas as atividades de vida diárias avaliadas, e a máxima de 100 equivale à independência total para as mesmas. Em relação ao índice de Barthel, os idosos foram classificados em duas categorias: dependentes ( 0 a 75 pontos) e independentes (acima de 75 pontos).

Durante o processo de coleta de dados, observou-se que alguns idosos cadeirantes, em determinadas situações, como alcançar um objeto ao alto ou ficar em ortostatismo para a troca de fraldas, tinham o potencial de não utilizar a cadeira de rodas. Assim, primeiramente esta questão foi confirmada através de consulta às enfermeiras do local. Posteriormente, o número de idosos com este potencial foi registrado. Estes idosos foram questionados sobre os fatores que levavam à utilização da cadeira, com a seguinte pergunta: "Por que motivo você utiliza a cadeira de rodas?” Os motivos relatados foram registrados.

Os dados foram armazenados e analisados com o auxílio do pacote estatístico Statistical Package for Social Sciences (SPSS), versão 21.0. Inicialmente, as variáveis foram analisadas descritivamente por meio de frequências absolutas e relativas (variáveis categóricas) e medidas de tendência central e dispersão (variáveis numéricas).

Os critérios paramétricos de normalidade e homogeneidade de variâncias foram analisados pelo teste de normalidade de Shapiro-Wilk. As variáveis idade, tempo de admissão, cognição e funcionalidade apresentaram distribuição normal, então, a comparação entre os grupos deu-se pelo teste $t$ de Student para amostras independentes.

$\mathrm{Na}$ análise bivariada foi utilizado o teste quiquadrado $\left(\chi^{2}\right)$ para a associação entre as exposições e a presença do uso de cadeira de rodas (desfecho). As medidas de associação, razão de chances (OR) e IC95\%, foram estimadas por meio da regressão logística. Adotou-se um nível de significância de $5 \%$.

\section{Resultados}

Foram incluídos no estudo um total de 55 idosos. O grupo G1 foi composto por 33(60\%) idosos, 15 homens e 18 mulheres e o grupo G2 por 22(40\%) idosos, 7 homens e 15 mulheres. Não houve diferença significativa entre os grupos em relação à idade $(p=0,549)$, tempo de admissão ( $p$ $=0,158)$ e cognição $(p=0,241)$. Houve diferença estatisticamente significativa entre os grupos em relação à funcionalidade $(p=0,005)$, onde a pontuação do Índice de Barthel foi inferior no G2. Dados representados na Tabela 1.

Os fatores idade, sexo, escolaridade, hipertensão, diabetes, dislipidemia e cognição não estão associados ao uso de cadeira de rodas. $\mathrm{O}$ idoso que não tem acidente vascular encefálico tem 0,09 menos chances de utilizar cadeira de rodas. A análise bruta da variável funcionalidade não pôde ser realizada, pois todos os cadeirantes eram dependentes nas atividades de vida diárias. Dados representados na Tabela 2.

Dos 22 idosos cadeirantes, 9 (41\%) tinham o potencial de não utilizar este dispositivo. Os fatores associados ao uso de cadeira de rodas relatados pelos idosos que apresentaram este potencial incluíram: medo de cair, dor, cansaço, fraqueza nas pernas, dificuldades de caminhar e falta de pessoas para auxiliar durante a deambulação. 
Tabela 1. Comparação entre os grupos em relação aos fatores analisados. Araranguá (SC), 2015.

\begin{tabular}{lrrr}
\hline & \multicolumn{1}{c}{ G1 } & \multicolumn{1}{c}{ G2 } & \multicolumn{1}{c}{ p } \\
\hline Idade & $74,3 \pm 8,8(71,2-77,4)$ & $76,0 \pm 8,5(72,2-79,7)$ & 0,549 \\
Tempo de admissão & $66,2 \pm 79,3(38,0-94,3)$ & $50,0 \pm 76,8(15,9-84,1)$ & 0,158 \\
Cognição & $14,5 \pm 8,5(11,4-17,5)$ & $10,8 \pm 6,5(7,9-13,7)$ & 0,241 \\
Funcionalidade & $80,3 \pm 22,7(72,2-88,4)$ & $11,4 \pm 14,3(5,0-17,7)$ & $0,005^{*}$ \\
\hline
\end{tabular}

Legenda: Dados expressos por média, desvio padrão e IC95\%. G1: locomoção sem auxílio, G2: cadeirantes. *Diferença estatisticamente significativa para $\mathrm{p}<0,05$.

Tabela 2. Análise da associação entre as exposições e a presença do uso de cadeira de rodas. Araranguá (SC), 2015.

\begin{tabular}{|c|c|c|c|c|c|c|}
\hline & \multirow[t]{2}{*}{ Variável } & \multicolumn{2}{|c|}{ Cadeira de rodas } & \multirow{2}{*}{ Total (\%) } & \multirow{2}{*}{ Valor de p } & \multirow{2}{*}{ OR bruto (IC95\%) } \\
\hline & & Não (\%) & $\operatorname{Sim}(\%)$ & & & \\
\hline \multirow[t]{3}{*}{ Idade } & & & & & 0,378 & \\
\hline & 75 anos ou menos & $19(65,5)$ & $10(34,5)$ & $29(52,7)$ & & 1 \\
\hline & Maior de 75 anos & $14(53,8)$ & $12(46,2)$ & $26(47,3)$ & & $1,62(0,54-4,82)$ \\
\hline \multirow[t]{3}{*}{ Sexo } & & & & & 0,312 & \\
\hline & Homens & $15(68,2)$ & $7(31,8)$ & $22(40,0)$ & & 1 \\
\hline & Mulheres & $18(54,5)$ & $15(45,5)$ & $33(60,0)$ & & $1,78(0,57-5,52)$ \\
\hline \multicolumn{2}{|c|}{ Escolaridade } & & & & 0,509 & \\
\hline & Não escolarizado & $15(55,6)$ & $12(44,4)$ & $27(49,0)$ & & 1 \\
\hline & Escolarizado & $18(64,3)$ & $10(35,7)$ & $28(51,0)$ & & $0,69(0,23-2,05)$ \\
\hline \multirow[t]{3}{*}{ AVE } & & & & & $0,000^{\star}$ & \\
\hline & Sim & $4(23,5)$ & $13(76,5)$ & $17(31,0)$ & & 1 \\
\hline & Não & $29(76,3)$ & $9(23,7)$ & $38(69,0)$ & & $0,09(0,02-0,36)$ \\
\hline \multicolumn{2}{|c|}{ Hipertensão } & & & & 0,252 & \\
\hline & Sim & $23(65,7)$ & $12(34,3)$ & $35(63,6)$ & & 1 \\
\hline & Não & $10(50,0)$ & $10(50,0)$ & $20(36,4)$ & & $1,91(0,62-5,87)$ \\
\hline \multirow[t]{3}{*}{ Diabetes } & & & & & 0,335 & \\
\hline & Sim & $8(72,7)$ & $3(27,3)$ & $11(20,0)$ & & 1 \\
\hline & Não & $25(56,8)$ & $19(43,2)$ & $44(80,0)$ & & $2,02(0,47-8,68)$ \\
\hline \multicolumn{2}{|c|}{ Dislipidemia } & & & & 0,312 & \\
\hline & Sim & $10(71,4)$ & $4(28,6)$ & $14(25,4)$ & & 1 \\
\hline & Não & $23(56,1)$ & $18(43,9)$ & $41(74,6)$ & & $1,95(0,52-7,27)$ \\
\hline \multicolumn{2}{|c|}{ Funcionalidade } & & & & $0,000^{*}$ & \\
\hline & Independente & $22(100,0)$ & $0(0,0)$ & $22(40,0)$ & & - \\
\hline & Dependente & $11(33,3)$ & $22(66,7)$ & $33(60,0)$ & & \\
\hline \multirow[t]{3}{*}{ Cognição } & & & & & 0,375 & \\
\hline & Normal & $16(66,7)$ & $8(33,3)$ & $24(43,6)$ & & 1 \\
\hline & Com declínio & $17(54,8)$ & $14(45,2)$ & $31(55,4)$ & & $1,64(0,54-4,97)$ \\
\hline
\end{tabular}

Legenda: Medidas de associação, (OR) e IC95\%, estimadas por meio da regressão logística * Diferença estatisticamente significativa para $\mathrm{p}<0,05$.

\section{Discussão}

Em instituições de longa permanência, onde os indivíduos são propensos a ter prejuízos mais graves e deficiências, o uso de dispositivos de mobilidade torna-se frequente ${ }^{5}$. Shields ${ }^{6}$ relata que de uma amostra de 22.500 idosos, aproximadamente $49 \%$ destes utilizavam cadeira de rodas. Clark et al. ${ }^{5}$ argumentam que de 294 idosos estudados, $54 \%$ faziam o uso deste dispositivo. Estes dados corroboram com o presente estudo, em que $40 \%$ da amostra utilizavam a cadeira de rodas. 
No atual estudo, não foi encontrada associação entre idade, sexo, escolaridade e cognição com o uso de cadeira de rodas. Clark et al..$^{5}$ apresentam dados semelhantes, verificando a não associação entre idade e cognição com o uso de cadeira de rodas. Entretanto, argumentam que idosos que apresentam menores recursos educacionais têm um maior risco de utilizar a cadeira de rodas.

Idosos com 65 anos de idade ou mais possuem uma maior chance de utilizar a cadeira de rodas $^{4,8}$. Além disso, os homens são mais propensos a essa utilização, pois as mulheres demonstram uma maior procura por serviços alternativos de assistência ${ }^{7}$.

Indivíduos que apresentam maiores dificuldades no desenvolvimento das atividades de vida diárias são mais propensos a utilizar a cadeira de rodas ${ }^{7}$. Pôde-se observar no atual estudo que a dependência nas atividades de vida diárias está associada à utilização de cadeira de rodas. Todos os cadeirantes desta pesquisa foram classificados como dependentes. Poucos estudos investigaram a relação entre o uso de cadeira de rodas e a capacidade funcional. Shields $s^{6}$ argumenta que aproximadamente $70 \%$ dos idosos cadeirantes estudados, necessitavam de assistência nas atividades básicas de autocuidado, principalmente em relação ao banho e vestimenta. Outras pesquisas também relatam esta relação, mas com foco em dispositivos de mobilidade em geral, chegando à mesma conclusão $0^{9,15}$.

O idoso com um quadro de acidente vascular encefálico apresenta maior risco para o uso de cadeira de rodas. Nenhum estudo analisou a associação entre a cadeira de rodas e as comorbidades especificamente (acidente vascular encefálico, hipertensão, diabetes e dislipidemia), mas apenas de forma conjunta. Clark e Colantonio ${ }^{7}$ averiguaram este fator em relação a idosos cadeirantes. Os mesmos descrevem que indivíduos com um maior número de condições crônicas de saúde são mais propensos a utilizarem a cadeira de rodas. Outros estudos confirmaram este achado, mas incluíram outros tipos de dispositivos em seus estudos (bengalas, muletas e andadores) ${ }^{9,15,16}$.

Idosos que sofreram acidente vascular encefálico apresentam agravos que implicam em pior desempenho para atividades que envolvam aspectos motores, pois há maior prejuízo da função muscular. Assim, muitas vezes estes necessitam de recursos e auxílio de terceiros para a execução destas atividades, principalmente em relação à locomoção ${ }^{17}$. Porém, mesmo que estes idosos desenvolvam limitações físicas decorren- tes do acidente vascular encefálico, podem apresentar um melhor desempenho, se houver maior investimento em prevenção, promoção à saúde e reabilitação, dentro das instituições de longa permanência ${ }^{18}$.

Clark e Colantonio ${ }^{7}$ abordam uma questão muito relevante. A literatura descreve que idosos com baixa aptidão funcional e um alto número de comorbidades, são mais propensos ao uso da cadeira de rodas. Entretanto, será que o uso deste dispositivo não poderia levar a maiores dificuldades para a realização das atividades de vida diárias e problemas de saúde adjacentes, em idosos que poderiam recorrer a outros tipos de recursos para a locomoção? Em função desta questão, o presente estudo considerou indispensável conhecer os fatores relatados pelos próprios idosos, já que os mesmos demonstravam certo potencial para a utilização de outros meios.

Estudo realizado em duas instituições de longa permanência descreve que fatores físicos poderiam estar relacionados com a decisão do idoso de utilizar ou não a cadeira de rodas ${ }^{11}$. Os resultados corroboram com os dados do atual estudo: dor, fraqueza muscular, baixa resistência e medo de cair. Entretanto, em instituições que oferecem meios alternativos de mobilidade, assistência pessoal, como também recursos reabilitacionais, como a fisioterapia, os idosos são menos propensos a utilizar a cadeira de rodas ${ }^{5}$. Gell et al. ${ }^{18}$ discutem que é imprescindível reduzir o índice de quedas em instituições de longa permanência, porém, buscando sempre manter o nível de capacidade funcional, que pode ser alcançado através da prescrição correta de dispositivos e da atuação ativa da fisioterapia nas instituições.

Muitas vezes, o que ocorre em uma instituição, com o intuito de agilizar o processo de atendimento, é a diminuição do incentivo aos idosos à realização independente das atividades, o que pode levar a uma piora do quadro funcional. Assim, cabe a toda equipe que atua nestas instituições, buscar desenvolver a autonomia, promovendo, consequentemente, uma melhor qualidade de vida a estes idosos ${ }^{19-21}$.

Crocker et al. ${ }^{22}$ apontam que programas de reabilitação têm o potencial de melhorar várias medidas da capacidade funcional de idosos residentes em instituição. $\mathrm{O}$ desempenho e a independência nas atividades de vida diárias, através das condutas fisioterapêuticas, são reforçados, ou declinam menos, em comparação com os cuidados habituais. Assim, pode-se evidenciar a necessidade de implantar a fisioterapia nestas instituições de longa permanência. 
Este estudo apresenta algumas limitações. A análise bruta da variável funcionalidade não pôde ser realizada, pois todos os cadeirantes eram dependentes nas atividades de vida diárias. Além disso, o tempo de admissão também não pôde ser incluído na análise, em vista da grande variação encontrada ( 1 a 372 meses) para uma amostra pequena.

Cabe salientar a necessidade de desenvolvimento de pesquisas com um maior número amostral, com o intuito de verificar novos fatores que possam estar associados ao uso de cadeira de rodas. O conhecimento destes fatores permitirá que novas alternativas de prevenção sejam traçadas.

\section{Colaboradores}

EF Alves foi a responsável pelo planejamento das idéias do artigo, elaboração da metodologia e pesquisa de dados para o embasamento da discussão. PP Bezerra auxiliou durante todo o processo de estruturação do artigo, análise dos dados estatísticos e desenvolvimento dos resultados.

\section{Conclusão}

Vários fatores podem influenciar um idoso institucionalizado a fazer o uso da cadeira de rodas. O acidente vascular encefálico e a funcionalidade são fatores que se associam a essa utilização. Para os idosos que apresentam o potencial de não utilizar este dispositivo, os motivos relatados são: medo de cair, dor, cansaço, fraqueza nas pernas, dificuldade de caminhar e falta de pessoas para auxiliar durante a deambulação. Muitos destes fatores podem ser prevenidos por meio de um maior apoio por parte da equipe de cuidadores, pela oferta de meios alternativos de mobilidade aos idosos e pela implantação da fisioterapia nestas instituições. O desenvolvimento de novas pesquisas pode permitir que alternativas de prevenção sejam traçadas, contribuindo para a redução do uso de cadeira de rodas por idosos institucionalizados. 


\section{Referências}

1. Instituto Brasileiro de Geografia e Estatística (IBGE). Atlas do censo demográfico 2010. Rio de Janeiro: IBGE; 2013.

2. Veras R. Fórum Envelhecimento populacional e as informações de saúde do PNAD: demandas e desafios contemporâneos. Cad Saude Publica 2007; 23(10):2463-2466.

3. Freedman VA, Martin, LG. Understanding functional limitations among older adults. Am J Public Health 1998; 88(10):1457-1462.

4. LaPlante M. Prevalence of mobility and self-care disability-United States, 1990. MMWR Morb Mortal Wkly Rep 1993; 42(39):760-761,767-768.

5. Clark P, Chan P, Santaguida PL, Colantonio A. The Use of Mobility Devices Among Institutionalized Older Adults. J Aging Health 2009; 21(4):611-626.

6. Shields M. Use of wheelchairs and other mobility support devices. Health Rep 2004; 15(3):37-41.

7. Clarke P, Colantonio A. Wheelchair Use Among Community-Dwelling Older Adults: Prevalence and Risk Factors in a National Sample. Can J Aging 2005; 24(2):191-198

8. Kaye HS, Kang T, LaPlante MP. Mobility device use in the United States. Disability Statistics Report (N. 14). Washington: Department of Education, National Institute on Disability and Rehabilitation Research; 2000.

9. Mathieson KM, Kronenfeld JJ, Keith VM. Maintaining functional independence in elderly adults: The roles of health status and financial resources in predicting home modifications and use of mobility equipment. Gerontologist 2002; 42(1):24-31.

10. Tomita M, Mann W, Fraas L, Burns L. Racial difference of frail elders in assistive technology. Assist Technol 1997; 9(2):140-151.

11. Pawlson LG, Goodwin M, Keith K. Wheelchair use by ambulatory nursing home residents. J Am Geriatr Soc 1986; 34(12):860-864.

12. Verbrugge LM, Rennert C, Madans JH. The great efficacy of personal and equipment assistance in reducing disability. Am J Public Health. 1997; 87(3):384-392.

13. Brucki SMD, Nitrini R, Caramelli P, Bertolucci PHR, Okamoto IH. Sugestões para o uso do mini-exame do estado mental no Brasil. Arq Neuropsiquiatr 2003; 61(3):777-781.

14. Mahoney FI, Barthel DW. Functional evaluation: the Bathel Index. Md. State Med. J 1965; 14:56-61.
15. Tomita MR, Mann WC, Fraas LF, Stanton KM. Predictors of the use of assistive devices that address physical impairments among community based frail elders. $J$ Appl Gerontol 2004; 23(2):141-155.

16. Gitlin LN, Schemm RL, Landsberg L, Burgh D. Factors predicting assistive device use in the home by older people following rehabilitation. J Aging Health 1996; 8(4):554-575.

17. Dantas CMHL, Bello FA, Barreto KL, Lima LS. Capacidade funcional de idosos com doenças crônicas residentes em Instituições de Longa Permanência. Rev Bras Enferm. 2013; 66(6):914-920.

18. Gell NM, Wallace RB, LaCroix AZ, Mroz TM, Patel KV. Mobility Device Use in Older Adults and Incidence of Falls and Worry About Falling: Findings from the 2011-2012 National Health and Aging Trends Study. J. Am. Geriat. Soc. 2015; 63(5):853-859.

19. Valcarenghi RV, Santos SSC, Barlem ELD, Pelzer MT, Gomes GC, Lange C. Alterações na funcionalidade/ cognição e depressão em idosos institucionalizados que sofreram quedas. Acta Paul Enferm 2011; 24(6):828833.

20. Alencar MA, Bruck NNS, Pereira BC, Câmara TMM, Almeida RS. Perfil dos idosos residentes em uma instituição de longa permanência. Rev. Bras. Geriatr. Gerontol. 2012; 15(4):785-796.

21. Davim RMB, Torres GV, Dantas SMM, Lima VM. Estudo com Idosos de Instituições Asilares no Município de Natal/RN: características socioeconômicas de saúde. Rev. Latino-Am. Enfermagem 2004; 12(3):518-524.

22. Crocker T, Forster A, Young J, Brown L, Ozer S, Smith J, Green J, Hardy J, Burns E, Glidewell E, Greenwood DC. Physical rehabilitation for older people in longterm care (Review). Cochrane Database Syst Rev 2013. Issue 2.

Artigo apresentado em 17/03/2016

Aprovado em 07/07/2016

Versão final apresentada em 09/07/2016 
